\title{
Reduction and Block Staining of Human Hair Shafts and Insect Cuticles by Ammonium Thioglycolate to Enhance Transmission Electron Microscopic Observations
}

\author{
Shigeru Sato ${ }^{1}$, Yoshihiro Sasaki ${ }^{1}$, Akiko Adachi ${ }^{1}$, Tokuya Omi ${ }^{2 *}$ \\ ${ }^{1}$ Division of Morphological and Biomolecular Research, Graduate School of Medicine, Nippon Medical School, Tokyo, Japan; \\ ${ }^{2}$ Department of Dermatology, Nippon Medical School, Tokyo, Japan. \\ Email: *t.omi@queens-sq.or.jp
}

Received February $19^{\text {th }}, 2013$; revised March $20^{\text {th }}, 2013$; accepted March $27^{\text {th }}, 2013$

Copyright (C) 2013 Shigeru Sato et al. This is an open access article distributed under the Creative Commons Attribution License, which permits unrestricted use, distribution, and reproduction in any medium, provided the original work is properly cited.

\begin{abstract}
We have developed a method where, after glutaraldehyde fixation, human hair shafts and insect cuticles are treated with ammonium thioglycolate (ATG) to improve ultrastructural staining. Conventional transmission electron microscopic (TEM) preparations do not distinguish the A-layer and the exocuticles of hair shafts. However, after ATG treatment, the A-layer appears in higher contrast. ATG treatment has also been used to observe the fibrillar structure in the cortex. In the cuticle of beetles, the epicuticle is stained by ATG. Although the human hair shaft (keratin) and insect cuticle (chitin) are composed of different materials, both can be reduced by the ATG solution. The ammonium in the ATG solution reacts with hair shafts and insect cuticles, causing a reduction of swelled cuticles. Therefore, ATG not only stains, but also reduces human hair shafts and the cuticles of beetles.
\end{abstract}

Keywords: Reduction and Block Staining; Ammonium Thioglycolate; Human Hair; Insect Cuticle; Electron Microscopy

\section{Introduction}

For wool, 2-mercaptoethanol (also known as thioglycol) in phosphate buffer is used for electron microscopic observations, called the reduction-osmication method. This method has proven to be the most successful for visualizing the fibrillar structures in the cortex [1-3]. When ammonium thioglycolate (ATG) solution is used for permanent waving of human hair shafts, changes in the physical and mechanical properties of human hair shafts occur, including a reduction in tensile strength the same as occurs with 2-mercaptoethanol [4-5]. The fibrillar structures in the cortex are obscured by conventional TEM. However, treatment with ATG or 2-mercaptoethanol in phosphate buffer has been used to observe the fibrillar structure in the cortex. In addition, the A-layer in hair cuticle cells is also stained [6-7].

Hair shafts are fully keratinized tissues that are difficult to prepare for histology (fixation, dehydration, embedding and sectioning). However, following treatment with ATG, the characteristics of hair shafts are changed

${ }^{*}$ Corresponding author. which makes them easy to prepare for TEM. It is a fact that after treatment with ATG, the hair shaft is reduced.

On the other hand, beetles have a hard cuticle due to their chitin composition [8-9]. The elytron of an adult beetle is too highly a sclerotized tissue to prepare for TEM embedding or sectioning. However, after treatment with ATG reduction, we found that the elytron is also reduced the same as hair shafts. The purpose of this study was to use ATG for TEM preparations to examine hair shafts and the hard cuticles of adult beetles. Further, we examined block staining with ATG solution to compare hair shafts and insect cuticles.

\section{Materials and Methods}

Healthy and chemically untreated hair shafts were taken from a Japanese female 18 years of age. The elytron and epidermis of an abdomen was taken from an adult male rhinoceros beetle, Trypoxylus dichotomus $\mathrm{L}$.

These specimens were fixed in $2.5 \%$ glutaraldehyde in $0.1 \mathrm{M}$ phosphate buffer at room temperature for $1-2 \mathrm{~h}$. The specimens were treated with $10 \%$ ATG solution $(\mathrm{pH}$ 9.5) in $0.1 \mathrm{M}$ phosphate buffer at room temperature for 1 
- $12 \mathrm{~h}$. After the specimens were completely reduced and were easily pliable they were washed multiple times in $0.1 \mathrm{M}$ phosphate buffer until the odor of ammonium disappeared and they were then post-fixed in $1 \%$ osmium tetroxide, dehydrated in a graded series of alcohol and embedded in Epon 812. Initially, semi-thin sections were cut $(0.5 \mu \mathrm{m}$ in thickness) with an ultramicrotome, and were stained with toluidine blue for light microscopic observations. For TEM, ultra-thin sections were cut with a diamond knife, were stained with uranyl acetate and lead citrate, and then were examined using a Hitachi H7650 transmission electron microscope. Untreated human hairs and beetle cuticles were also examined by conventional TEM.

\section{Results}

\subsection{Human Hair Shafts}

Seven or eight over-lapping cuticle cells occur in human hair, each with an intact A-layer (Figures 1 and 2). In conventional TEM, two layers were seen in each cuticle cell and the outer layer was electron lucent. In the inner layer, endocuticles are more electron dense than in the outer layer (Figure 1(A)). Block staining with ATG solution produced a higher contrast of the A-layer and more contrast of the exocuticle than the endocuticle (Figure

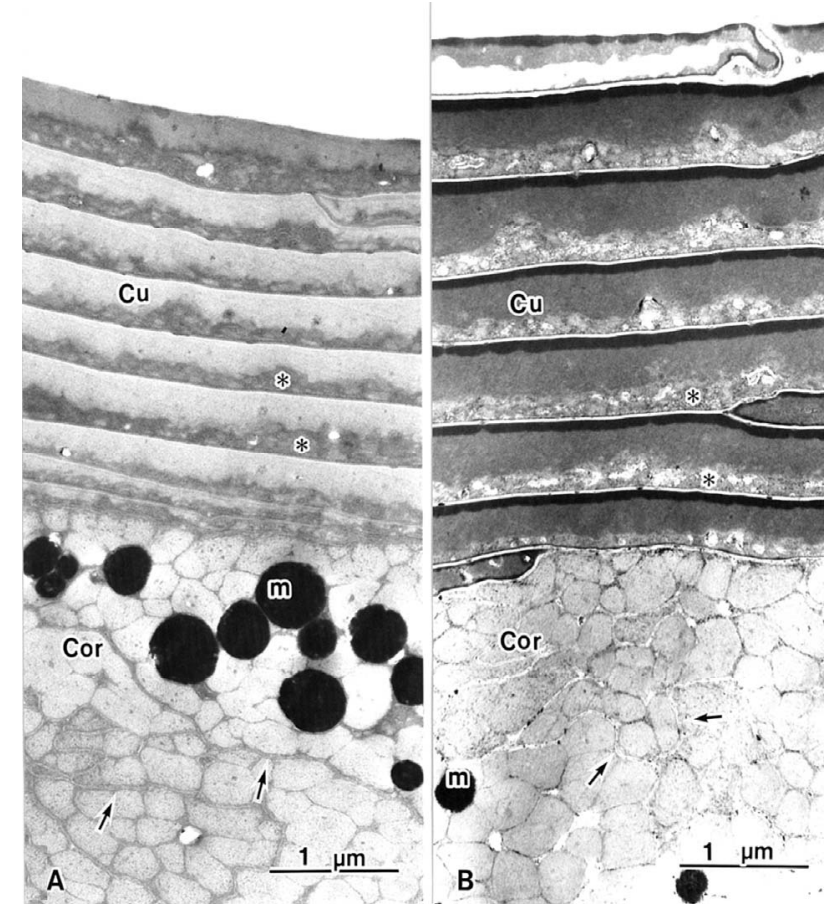

Figure 1. Hair shaft showing eight or nine overlapping cuticle cells $(\mathrm{Cu})$. The cortex (Cor) has cortical cells. (A) A-layers are obscured in conventional TEM; (B) A-layers are stained by ATG treatment. m: melanin granule; asterisk: endocuticle; arrow: trilaminal cell membrane.
1(B)). The A-layer and exocuticle layers were homogenous. The endocuticle layer showed a variously sized granule-like structure (Figures $\mathbf{1}$ and 2). The cell membrane complex (Figures $\mathbf{1}$ and $\mathbf{2}$ arrows) consists of two electron-lucent layers (the $\beta$ bands) and a central electron-dense layer (the $\delta$ band), which are clearly seen after ATG staining more than by conventional TEM between each cuticle cell (Figure 2 arrows). ATG-treated cuticle cells were about 1.5 times as thick as the untreated cuticle cells (Figure 1).

The cortex cells contained many macrofibrils (Figure 1). Conventional TEM showed that each macrofibril was identified by an intermacrofibrillar matrix, but the microfibrils were obscured in a macrofibril (Figure 3(A)). Block staining with the ATG solution produced a higher contrast of the intermicrofibrillar matrix in cross-sections of hair shafts. Electron lucent microfibrils were also clearly seen in macrofibrils due to the electron dense intermicrofibrillar substance. The axial microfibril bundle was surrounded by concentric lamellae of microfibrils, showing a fingerprint appearance. The diameter of each microfibril was 7 - $9 \mathrm{~nm}$ (Figure 3(B)).

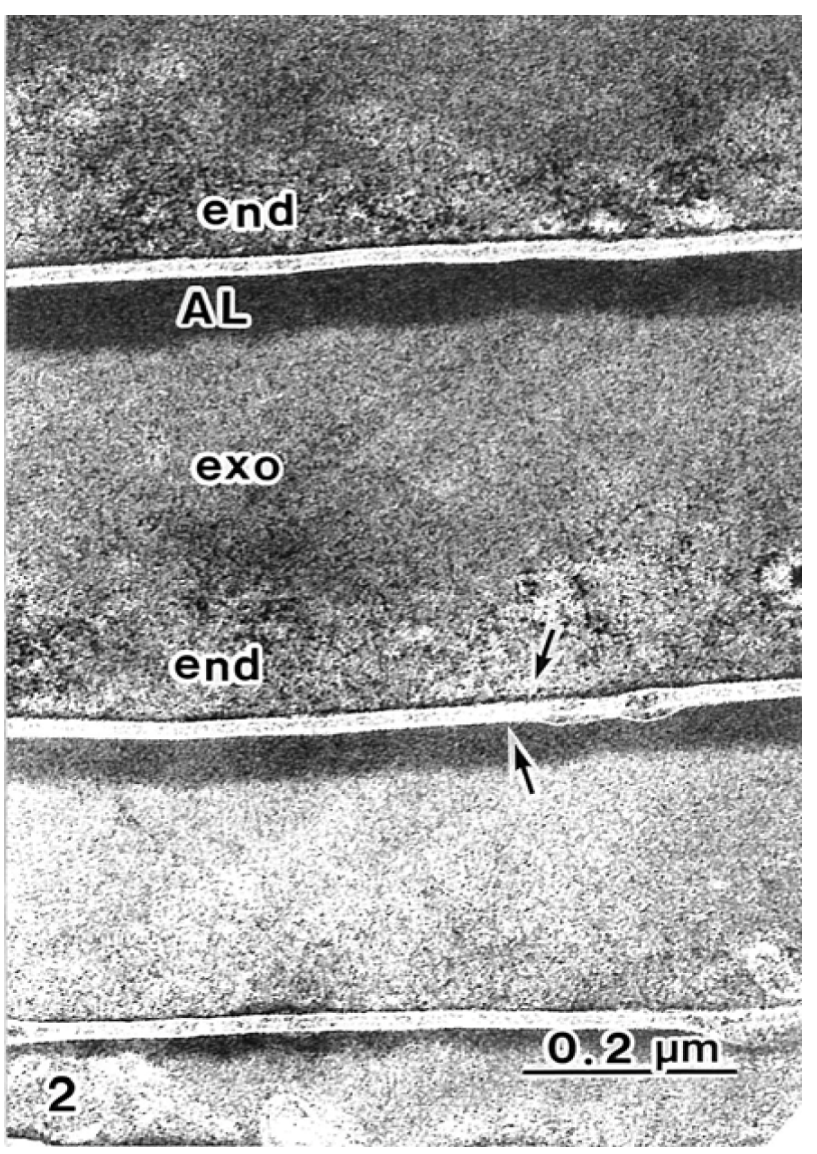

Figure 2. A-layers (AL) and exocuticles (exo) in the hair shaft are stained by ATG. Each cuticle cell is intact with trilaminal cell membranes (arrows). 


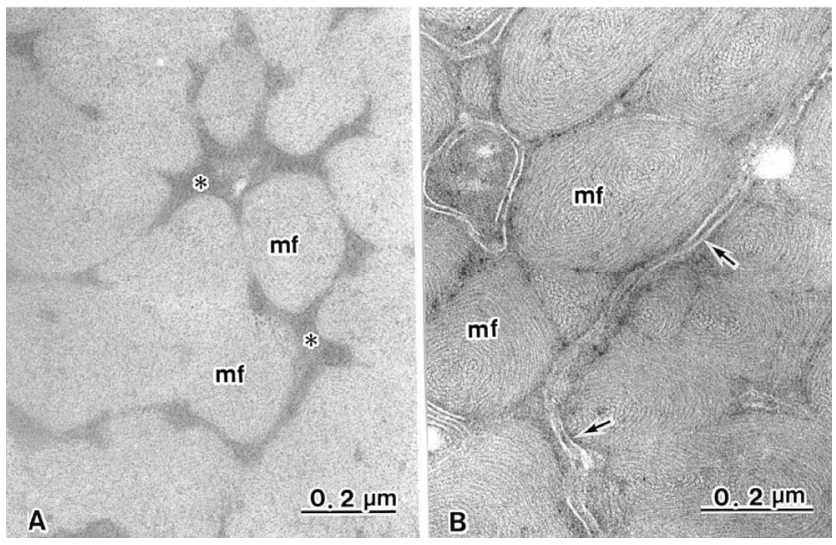

Figure 3. Cortical cells contain many macrofibrils (mf), each intact with trilaminal cell membranes (arrows). (A) Intermacrofibrillar matrix (asterisks) is seen in non-treated ATG cortex. Fine structures of microfibrils in macrofibrils are obscured; (B) Intermacrofibrillar matrix is not seen. Macrofibrils are composed of whorl-like arrangements of microfibrils following ATG treatment.

\subsection{Cuticle of the Rhinoceros Beetle}

In insects, the cuticle is secreted by epidermal cells. After ATG treatment, cuticles of both the elytron and the abdomen were the same thickness (about $70 \mu \mathrm{m}$ ). In the elytron, the epicuticle is a thin layer, highly stained by toluidine-blue (Figure 4(A)). The exocuticle is stained light blue, and the endocuticle is stained dark blue in the elytron and the abdomen (Figures 4(A) and (B)). In the abdomen cuticle, both the exocuticle and the endocuticle are the same thickness as the elytron cuticle. The epicuticle is obscured by the thin layer in the abdomen (Figure 4(B)).

Block staining with ATG solution produced a higher contrast of epicuticles (Figures 5 and 6(B)) compared with the routine TEM method (Figure 6(A)). Epicuticles in the elytron (about $3 \mu \mathrm{m}$ in thickness) were thicker than the abdomen epicuticles (about $0.5 \mu \mathrm{m}$ in thickness) (Figures 4 and 5). Each lamella of the exocuticle under the epicuticle, was about $0.2 \mu \mathrm{m}$ thick in the elytron, (Figure 5), and $0.9-1.0 \mu \mathrm{m}$ thick in the abdomen. The lamellae are composed of microfibrils, about 70 layers in the elytron exocuticle (Figure 5), and about 12 - 14 layers in the abdomen exocuticle (Figure 6). The helicoidal microfibrils of each lamella were evident in the exocuticle (Figure 6), but non-helicoidal hyaline microfibrils were seen in the endocuticle (Figure 7). Each lamella of the endocuticle in both non-treated and ATG-treated specimens was about $3 \mu \mathrm{m}$ in thickness. Epidermal cells had no ultrastructural changes following ATG treatment (Figure 7). Following ATG treatment, each lamella (0.7 $\mu \mathrm{m}$ in thickness) in the exocuticle was 1.4 times as thick as the untreated abdomen exocuticle (Figure 6). However, the thickness of the endocuticle was not changed by

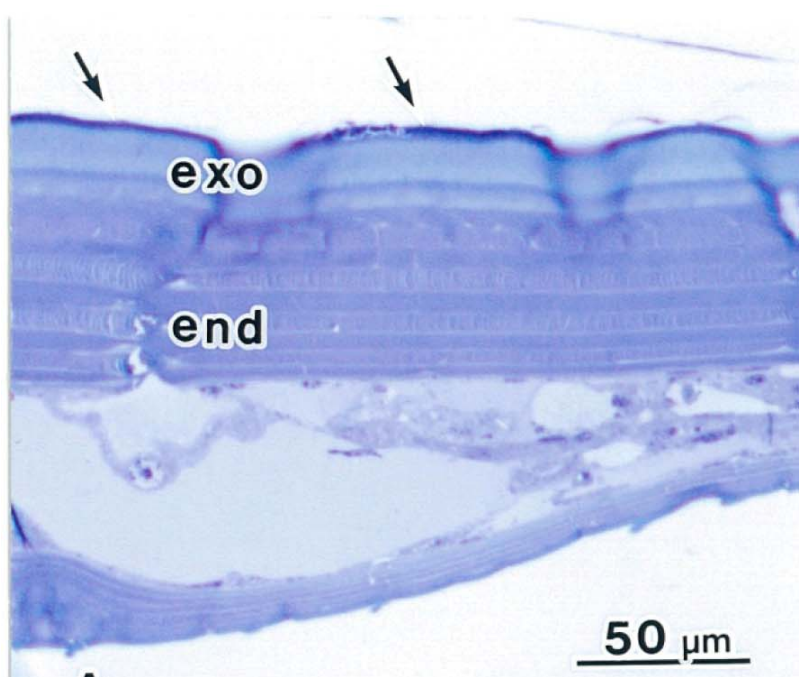

A

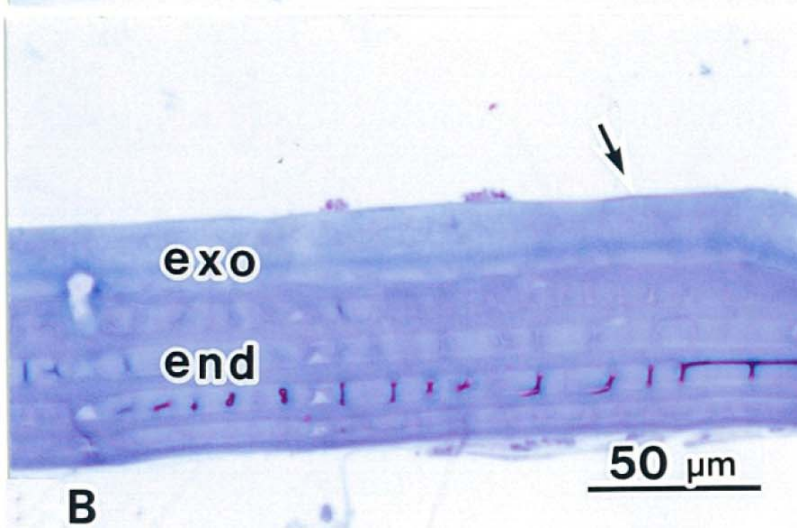

Figure 4. Light micrographs showing cuticles in a rhinoceros beetle. The epicuticle is a thin layer, highly stained (arrows). The exocuticle (exo) is light blue. The endocuticle (End) is dark blue colored by toluidine-blue. (A) Elytron following ATG treatment; (B) Abdomen following ATG treatment.

\section{ATG treatment.}

\section{Discussion}

\subsection{Block Staining}

Conventional TEM showed no distinction between the A-layer and the exocuticle in the hair shaft. However, after cuticles were treated with ATG, the A-layer and the exocuticle could be distinguished by the stained A-layer. The fibrillar structure in the cortex is also obscured in conventional TEM, but ATG treatment allows the fibrillar structure to be observed. Each electron lucent microfibril is clearly observed in a microfibril caused by staining of an intermicrofibrillar amorphous matrix [1-3, 7,10]. After glutaraldehyde fixation, human hair shafts and beetle cuticles were treated with the ATG solution, and were observed by TEM. In the cuticle of the beetle, 


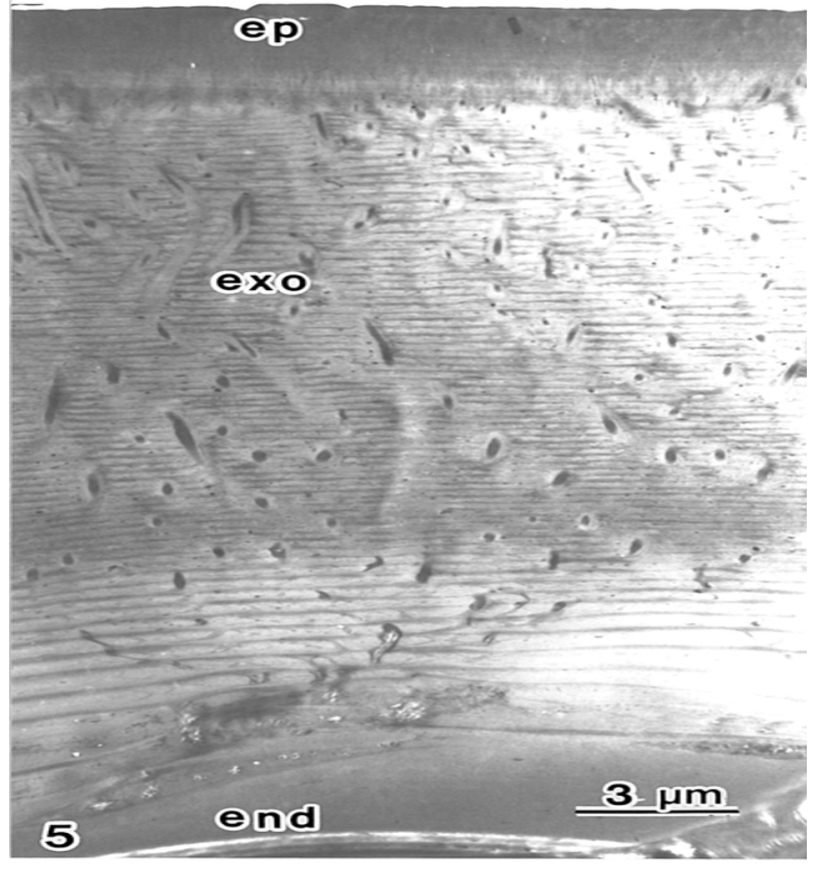

Figure 5. The cuticle in the beetle elytron is composed of an epicuticle (ep) an exocuticle (exo) and an endocuticle (end) following ATG treatment. Seventy layers of lamellae are seen in the exocuticle.

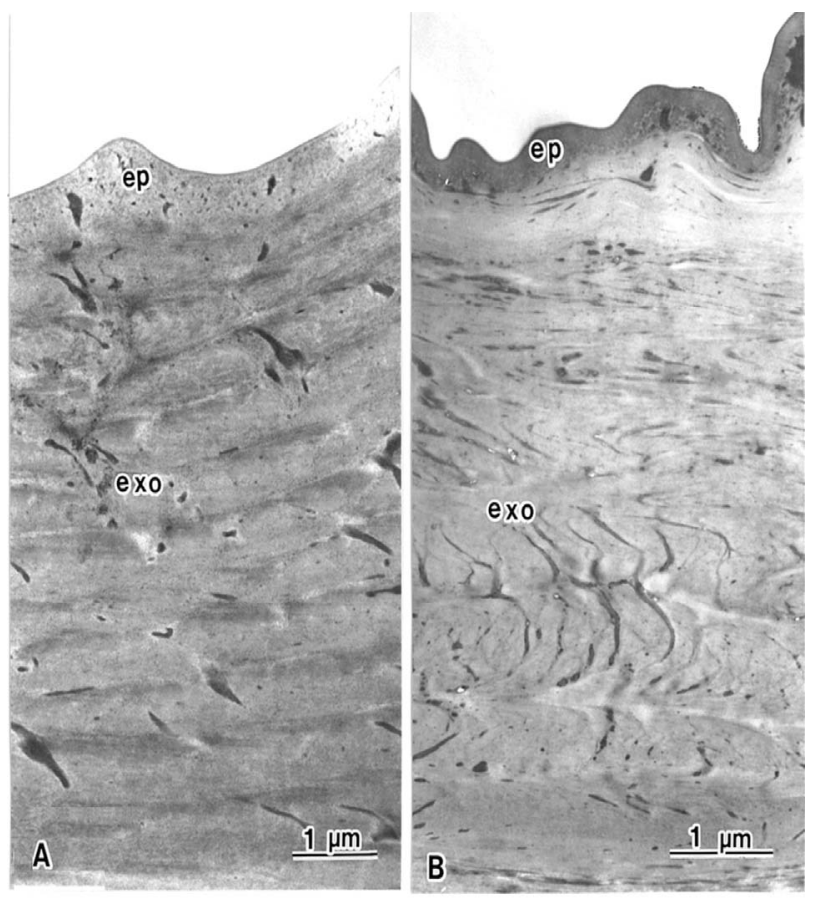

Figure 6. The epicuticle (ep) of the beetle is the outermost layer in the abdomen epidermis, and is not composed of lamellae structures. The exocuticle is composed of lamellae structures. (A) Epicuticle is electron lucent in conventional TEM. Each lamella is $0.5 \mu \mathrm{m}$ thick; (B) Epicuticle is electron dense following ATG treatment. Each lamella is $0.7 \mu \mathrm{m}$ thick.

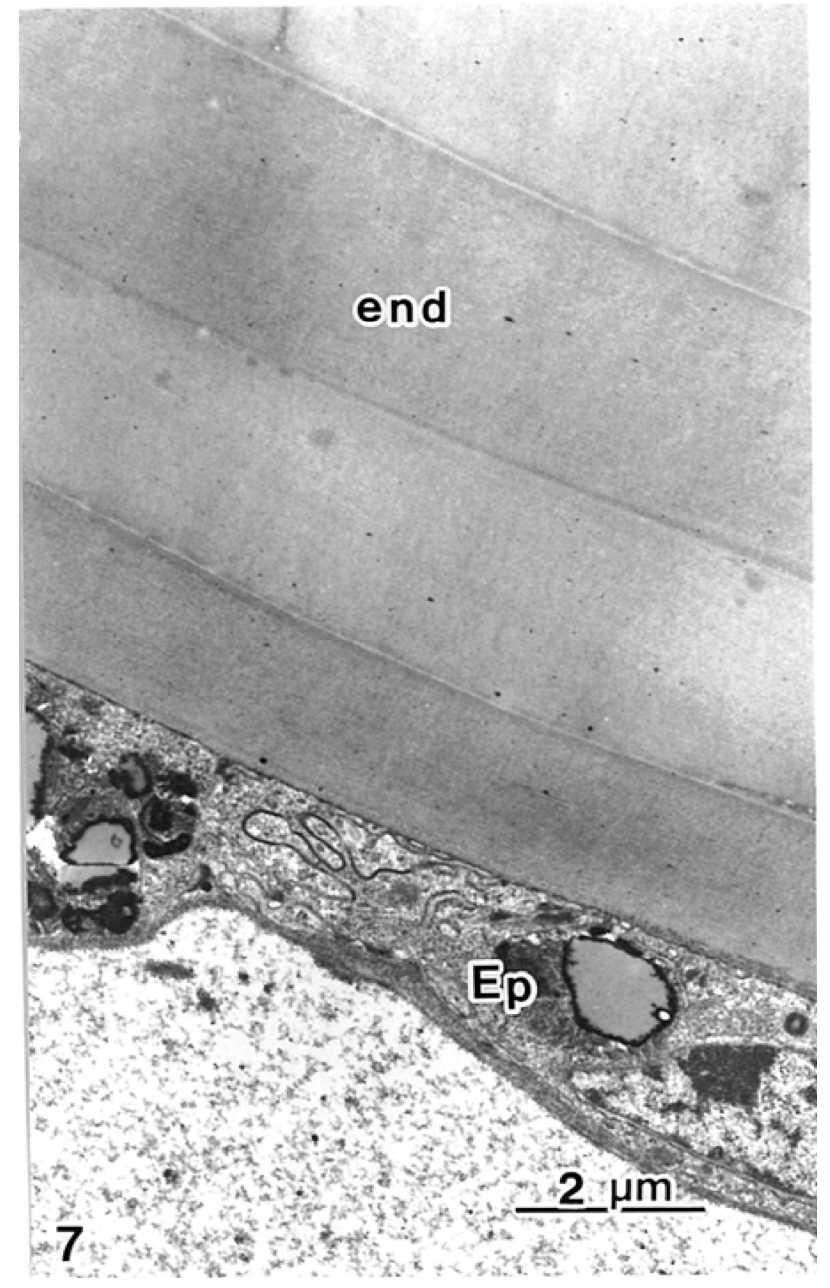

Figure 7. Endocuticle and epidermal cells are seen.

the epicuticle was stained by ATG. Epicuticles include lipids and minerals in comparison with other cuticles [11]. We hypothesize that lipids and/or minerals are stained by thioglycolate. However, the composition of the A-layer in the hair cuticle is not known to include lipids or minerals. On the other hand, the A-layer of cuticle cells is particularly resistant both to trypsin digestion and to keratinolytic attack [12]. The epicuticle of the insect is also the outermost layer, as is the A-layer, and has waterproofing properties, resistance to fungi and some chemicals, etc. If the anti-resistance materials are stained by thioglycolate, it is very interesting.

\subsection{Reduction}

Ammonium thioglycolate (ATG) solution is used for permanent waving of human hair, and changes in the physical and mechanical properties of human hair are caused by a reduction in tensile strength [4,5]. ATGtreated cuticle cells are swollen, and are 1.5 times as thick compared with untreated cuticle cells. Hair shafts 
following ATG treatment not only have swollen cuticle cells, but also have swollen macrofibrils [6]. However, swollen macrofibrils were not detected in this study. In general, the reduction in tensile strength caused by treatment with ATG is caused by the cleavage of the S-S bond in keratin fibers $[1,13]$. However, it has been reported that S-S bond intensity that exists in the cortex is not changed by ATG treatment [14].

On the other hand, after treatment with ATG solution, we found that the elytron of beetles is also reduced as are the hair shafts. In this study, exocuticles treated with ATG exocuticles are swollen, but epi- and endo-cuticles are not. Insect exo- and endo-cuticles are composed of chitin, a nitrogen-containing polysaccharide. In addition, the elytron in conventional TEM could not be cut in ultra-thin sections. In spite of hair shafts and insect cuticles being composed of different materials, both are reduced by the ATG solution. It may be that the ammonium of the ATG solution reacts with human hair shafts and insect cuticles. In fact, Harland et al. [7] showed that treatment with 2-mercaptoethanol in phosphate buffer causes cuticle cells in human hair to be about $0.4 \mu \mathrm{m}$ in thickness, but, by using the ammonical silver method, cuticle cells are 1.5 times thicker. Thus, the ammonium of the ATG solution reacts both with hair shafts and with insect cuticles and causes swelling of human cuticle cells and insect exocuticles.

\section{REFERENCES}

[1] G. E. Rogers, "Electron Microscopy of Wool," Journal of Ultrastructure Research, Vol. 2, No. 3, 1959, pp. 309330. doi:10.1016/S0022-5320(59)80004-6

[2] D. F. Orwin, J. L. Woods and S. L. Ranford, "Cortical Cell Type and Their Distribution in Wool Fibres," Australian Journal of Biological Sciences, Vol. 37, No. 4, 1984, pp. 237-255.

[3] J. L. Woods, D. P. Harlamd, J. A. Vernon, G. L. Krsinic and R. J. Walls, "Morphology and Ultrastructure of Antler Velvet Hair and Body Hair from Red Deer (Cervus Elaphus)," Journal of Morphologyl, Vol. 272, No. 1, 2011, pp. 34-49. doi:10.1002/jmor.10893

[4] R. Beyak, C. F. Meyer and G. S. Kass, "Elasticity and
Tensile Properties of Human Hair I. Single Fiber Test Method," Journal of the Society of Cosmetic Chemists, Vol. 20, No. 10, 1969, pp. 615-620.

[5] F. J. Wortman and I. Souren, "Extensional Properties of Human Hair and Permanent Waving," Journal of the Society of Cosmetic Chemists, Vol. 38, No. 2, 1987, pp. 125-140.

[6] T. Takizawa, S. Arai, M. Osumi and T. Saito, "Ultrastructure of Human Scalp Hair Shafts as Revealed by Freeze-Substitution Fixation," The Anatomical Record, Vol. 251, No. 3, 1998, pp. 406-413. doi:10.1002/(SICI)1097-0185(199807)251:3<406::AIDAR17>3.0.CO;2-S

[7] D. P. Harland, J. A. Vernon, R. J. Walls and J. L. Woods, "Transmission Electron Microscopy Methods for the Cortex of Human Hair: A Modified Osmium Method and Comparison with Other Stains," Journal of Microscopy, Vol. 243, No. 2, 2011, pp. 184-196. doi:10.1111/j.1365-2818.2011.03493.x

[8] A. C. Neville D. A. D. Parry and J. Woodhead-Galloway, "The Chitin Crystallite in Arthropod Cuticle," Journal of Cell Science, Vol. 21, No. 1, 1976, pp. 73-82.

[9] K. F. Barry and N. F. Hadley, "Fine Structure of the Cuticle of the Desert Scorpion, Hadrurus Arizonesis," Tissue \& Cell, Vol. 11, No. 2, 1979, pp. 249-262. doi:10.1016/0040-8166(79)90040-5

[10] C. Orfanos and H. Ruska, "Die Feinstruktur des Menschlichen Haares; II. Der haar-Cortex," Archiv für Klinische und Experimentelle Dermatologie, Vol. 231, No. 3, 1968, pp. 264-278.

[11] V. B. Wigglesworth, "Incorporation of Lipid into the Epicuticle of Rhodnius (Hemiptera)," Journal of Cell Science, Vol. 19, No. 3, 1975, pp. 459-485.

[12] M. S. Birbeck and E. H. Mercer, "The Electron Microscopy of the Human Hair Follicle. The Hair Cuticle," Birbeck and Mercer, Vol. 3, No. 2, 1957, pp. 215-222. doi:10.1083/jcb.3.2.215

[13] C. R. Robbins, "Chemical and Physical Behavior of Human hair," 2nd Ediyion, Springer-Verlag, New York. 1988. doi:10.1007/978-1-4757-2009-9

[14] A. Kuzuhara, "Analysis of Structural Changes in Permanent Waved Human Hair Using Raman Spectroscopy," Biopolymers, Vol. 85, No. 3, 2006, pp. 274-283. doi:10.1002/bip.20646 